\title{
Robotic Total Mesorectal Excision for Rectal Cancer: Short-Term Oncological Outcomes of Initial 178 Cases
}

\author{
C. Ramachandra ${ }^{1} \cdot$ Pavan Sugoor $^{1}$ (D) $\cdot$ Uday Karjol $^{1} \cdot$ Ravi Arjunan $^{1} \cdot$ Syed Altaf ${ }^{1} \cdot$ C. Srinivas $^{1} \cdot$ B. V. Prakash ${ }^{1}$. \\ Vijay Patil ${ }^{1}$
}

Received: 20 May 2020 / Accepted: 3 September 2020 / Published online: 8 September 2020

(C) The Author(s) 2020

\begin{abstract}
Emerging techniques in minimally invasive rectal resection include robotic total mesorectal excision (R-TME). The Da Vinci Surgical System offers precise dissection in narrow and deep confined spaces and is gaining increasing acceptance during recent times. The aim of this study is to analyse our initial experience of R-TME with Da Vinci Xi platform in terms of perioperative and oncological outcomes in the context of data from recently published randomised ROLARR trial amongst minimally invasive novice surgeons. Patients who underwent R-TME or tumour specific mesorectal excision for rectal cancer between May 2016 and November 2019 were identified from a prospectively maintained single institution colorectal database. Demographic, clinical-pathological and short-term oncological outcomes were analysed. Of the 178 patients, $117(65.7 \%)$ and $31(17.4 \%)$ patients had lower and mid third rectal cancer. Most of the tumours were locally advanced, cT3-T4: 138 (77.5\%). One hundred/ $178(56.2 \%)$ underwent sphincter preserving TME. Eighty-seven (48.8\%) were grade II adenocarcinoma. Nonmucinous adenocarcinoma was the predominant histology, $138(78.4 \%)$. One hundred one cases $(56.7 \%)$ were pT3. The mean number of lymph node yield was $13 \pm 5$. Distal resection margin and circumferential resection margin were positive in $2(1.12 \%), 12$ cases $(6.74 \%)$ respectively. Eleven cases (6.7\%) had to be converted to open TME. Mean blood loss and duration of surgery was $170 \pm 60 \mathrm{ml}$ and $286 \pm 45 \mathrm{~min}$ respectively. Five percent cases had an anastomotic leak. Grade IIIa-IIIb Clavien Dindo (CD) morbidity score was reported to be in $12(6.75 \%)$ and $10(5.61 \%)$ cases. Median length of hospitalisation was 7 days (range 4-14 days). Perioperative and pathologic outcomes following robotic rectal resection is associated with good short-term oncological outcomes and is safe, effective, and reproducible by a minimally invasive novice surgeon.
\end{abstract}

Keywords Rectal cancer $\cdot$ Robotic rectal resection · Robotic total mesorectal excision $\cdot$ Da Vinci surgical system

\section{Introduction}

Neoadjuvant chemoradiotherapy (NACRT) has a major role in the treatment of locally advanced rectal tumours [1]. Oncological outcomes have improved following widespread acceptance of the principles of TME [2]. Surgical techniques govern oncological outcomes in rectal cancer surgery. Tumour-specific mesorectal excision or total mesorectal excision (TME) and achieving a negative circumferential resection margin (CRM) are associated with lower recurrence rates and improved overall survival [3-9].

Pavan Sugoor

pavansugoor26@gmail.com

1 Department of Surgical Oncology, Kidwai Memorial Institute of Oncology, Bengaluru, Karnataka, India
There have been numerous prospective randomized studies about the superior short-term outcomes of laparoscopic surgery for rectal cancer in comparison with open rectal resections $[10-14]$. Few studies have raised concerns on the quality of TME, composite pathological outcomes, and the oncological safety associated with the laparoscopic total mesorectal excision (L-TME approach) [15-17]. The question remains still open after the publication of the results of the latest trials [17-20].

Poor visibility coupled with difficult access in deep and narrow pelvis makes dissection and distal division a technical challenge, which may result in suboptimal pathological outcomes and hence are the fundamental concerns with L-TME. This has led some surgeons to adopt robotic-assisted approach which aims to improve the ease and quality of TME while still retaining the potential benefits of minimally invasive approach. 
Robotic-assisted approach offers magnified three dimensional vision, a surgeon-controlled camera and operating platform, instruments with various degrees of freedom and articulation, enhanced ergonomics and tremor filtration [21]; these advantages may translate to superior TME quality and improved autonomic functional outcomes [22-24].

ROLARR randomized trial, compared laparoscopic versus robotic surgery and reported robotic approach may have a lower overall conversion rate particularly benefiting obese men subgroup. The study did not find any statistical significant differences in the rest of the short-term outcomes including bladder and sexual dysfunction [25].

R-TME at our institution is being performed since 2016 and has become the preferred procedure of choice in patients who are suitable for a minimally invasive approach. The objective of this study was to prospectively evaluate the initial experience with R-TME and associated perioperative and oncologic outcomes in the context of data from recently published ROLARR trial.

\section{Materials and Methods}

Retrospective review of a prospectively maintained colorectal database identified 178 patients who had underwent robotic rectal resections using Da Vinci Xi Robotic system (Intuitive Surgical Inc., Sunnyvale, CA, USA) for biopsy confirmed primary rectal adenocarcinoma or melanoma at Kidwai Memorial Institute of Oncology, Bengaluru, India, between May 2016 to November 2019. Rectal cancer was defined as tumours that were $\leq 15 \mathrm{~cm}$ from the anal verge and were grouped into upper $(11-15 \mathrm{~cm})$, mid $(6-10 \mathrm{~cm})$, and lower $(\leq 5 \mathrm{~cm})$ based on distance from anal verge.

\section{Preoperative Staging and Neoadjuvant Treatment}

All patients underwent preoperative staging of pelvis with magnetic resonance imaging (MRI) and computed tomography (CT) scans of the abdomen and thorax. NACRT was offered to patients with clinical stage T3-T4 N0 or any T $\mathrm{N}+$ with or without mesorectal fascia (MRF) involvement. The NACRT regimen included oral capecitabine-based chemotherapy and external beam radiation (a total dose of 50.4 Gy in 25 fractions). After completion of NACRT, all patients underwent restaging pelvic MRI. If MRF/CRM remained positive, additional 4 cycles of capecitabine- and oxaliplatin-based chemotherapy were administered. Surgery was performed between 7 and 8 weeks postNACRT.

Early T3 (T3a-T3b) [26], CRM/MRF negative cases received short-course radiotherapy (SCRT: $25 \mathrm{~Gy}$ in 5 fractions) and underwent surgery between 3 and 7 days postSCRT. All patients received adjuvant chemotherapy for a period of 6 months.

\section{Eligibility Criteria}

Patients who underwent abdomen/pelvic dissection after docking were included in the analysis. Robotic surgery was not offered for patients requiring extended resection, complex abdominal wall reconstruction, or synchronous liver resection with laparotomy. Cases with presence of significant intraabdominal adhesions limiting access to the distal colon or pelvis or peritoneal deposits on staging/diagnostic laparoscopy were excluded.

\section{Outcome Assessment}

The primary measures of this analysis were perioperative and pathological outcomes. $<1 \mathrm{~mm}$ between deepest tumour extension to the CRM was defined as positive CRM while < $1 \mathrm{~mm}$ between the lowest aspect of tumour and distal cut edge of specimen was considered as a positive distal resection margin (DRM) [27].

\section{Defining Conversion}

Conversion to open surgery was defined as the use of an abdominal incision to continue the procedure under direct visualization before completion of the TME, due to any cause.

\section{Surgical Complications}

Any adverse events within and after 30 days after surgery were defined as postoperatively early and delayed complications respectively. Anastomosis leakage was defined as feculent discharge in surgically placed drains, radiological evidence of contrast extravasation or perianastomotic collection requiring drainage, or anastomotic dehiscence as determined on digital rectal examination or flexible sigmoidoscopy.

\section{Technique of Totally Robotic TME for Rectal Cancer}

In the present study, all patients underwent single docking, single stage and complete R-TME using the Da Vinci Surgical System Xi [28].

\section{Data Collection and Statistical Analysis}

All demographic, operative, pathological and postoperative recovery data were obtained from the prospectively maintained colorectal database. Surgical complications were stratified by Clavien Dindo classification system [29]. All statistical analyses were carried out with the Statistical Package for the Social Sciences version 21 (SPSS Chicago, IL, USA). Continuous variables were used to derive the mean $\pm \mathrm{SD}$ (SD, standard deviation). 


\section{Ethics}

The data of the present study were collected in the course of common clinical practice, and, accordingly, the signed informed consent was obtained from each patient for any surgical and clinical procedure. The study protocol conforms to the ethical guidelines of the World Medical Association Declaration of Helsinki: Ethical Principles for Medical Research Involving Human Subjects adopted by the 18th WMA General Assembly, Helsinki, Finland, June 1964, as revised in Tokyo 2004. No approval of the institutional review committee was needed.

\section{Results}

A total of 335 robotic resections were performed during the study period of which 178 cases underwent robotic rectal resection Table 1, Fig. 1. R-TME was performed initially by a single surgeon with 2 additional surgeons progressively transitioning from open to robotic during the study period with annual increase in the total number of cases performed robotically.

Table 1 Institutional database of robotic resections

\begin{tabular}{ll}
\hline Procedures & Numbers $(n)=335$ \\
\hline 1. Lower GI & 210 \\
a. R-TME & 178 \\
b. R-CME & 32 \\
2. Upper GI & 82 \\
a. TTE & 70 \\
b. Subtotal gastrectomy & 10 \\
c. Total gastrectomy & 02 \\
3. Genitourinary & 36 \\
a. Radical nephrectomy & 19 \\
b. RCIC & 10 \\
c. RARP & 06 \\
d. Adrenalectomy & 01 \\
4. Hepatobiliary & 06 \\
a. PPPD & 01 \\
b. DPS & 01 \\
c. Radical cholecystectomy & 01 \\
d. Simple cholecystectomy & 03 \\
5. Mediastinum and thorax & 01 \\
a. Thymoma excision & \\
\hline
\end{tabular}

$R$-TME Robotic total mesorectal excision, $R-C M E$ robotic complete mesocolic excision, $R C I C$ radical cystectomy with ileal conduit, $R A R P$ robotic-assisted radical prostatectomy, $P P P D$ pylorus preserving pancreatico-duodenectomy, DPS distal pancreatico-splenectomy

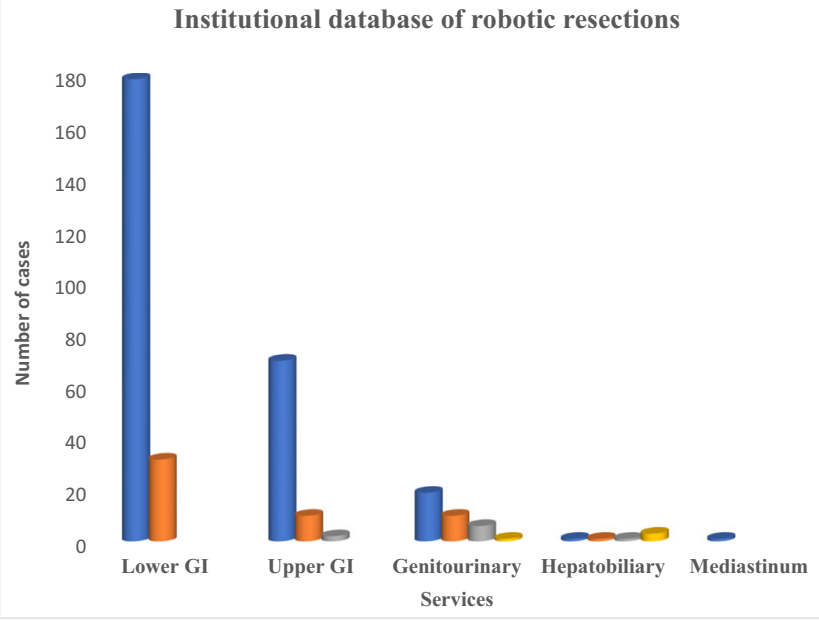

Fig. 1 Institutional database of robotic resections

\section{Patient Characteristics}

The demographic characteristics are summarized in Table 2. Median age was 51 years (range: $23-87$ years). $51.7 \%(n=$ $92)$ and $48.3 \%(n=86)$ were men and women respectively. Mean body mass index was $22.8 \pm 4$. Lower third and middle third rectal tumours amounted for $65.7 \%(n=117)$ and $17.4 \%$ $(n=31)$ cases respectively. Locally advanced T3-T4 tumours were predominant, accounted for $77.5 \%(n=138)$ of the cases. $65.5 \%$ received neoadjuvant therapy.

\section{Operative Outcomes}

Table 3 illustrates operative parameters. One hundred sixtyseven/178 underwent complete robotic rectal resections. Sphincter preservation procedures amounted for $56 \%(n=$ $100)$ of cases, and the remaining $44 \%(n=78)$ were Abdomino-perineal resections (APR). Mean total operating time, docking and surgeon console time were $286 \pm 45 \mathrm{~min}$, $13 \pm 5 \mathrm{~min}$ and $220 \pm 20 \mathrm{~min}$ respectively. Mean blood loss was $170 \pm 60 \mathrm{ml}$.

\section{Docking Time}

Mean docking time is $13 \pm 5 \mathrm{~min}$. Our analysis showed that with a standardised six port technique the docking time plateaus after 18 consecutive cases (Fig. 2).

\section{Time Stratified Analysis}

During the first year, the ratio of APR to sphincter preserving procedures was $4: 1$, with increasingly gaining experience the ratio was 2:2 during the first half of the second year and 1:4 thereafter (Fig. 3). 
Table 2 Baseline characteristics of the patients

\begin{tabular}{ll}
\hline Variables & Numbers $(n)$ \\
\hline 1. Median age & 51 years $(23-87$ years $)$ \\
2. Gender & \\
a. Male & $92(51.7 \%)$ \\
b. Female & $86(48.3 \%)$ \\
3. Mean BMI & $22.8 \pm 4$ \\
4. ASA & \\
a. I-II & $18(66.2 \%)$ \\
b. III-IV & $60(33.7 \%)$ \\
5. Tumour location & \\
a. Upper third & $30(16.9 \%)$ \\
b. Middle third & $31(17.4 \%)$ \\
c. Lower third & $117(65.7 \%)$ \\
6. Preoperative T stage & \\
a. T1-T2 & $40(22.4 \%)$ \\
b. T3-T4 & $138(77.5 \%)$ \\
7. Preoperative N stage & \\
a. Node negative & $64(35.9 \%)$ \\
b. Node positive & $114(64.0 \%)$ \\
8. Baseline CRM & \\
a. Free & $142(79.77 \%)$ \\
b. Involved & $36(20.24 \%)$ \\
a. Yeoadjuvant treatment & \\
b. No & $61(34.5 \%)$ \\
\hline &
\end{tabular}

An annual analysis of cases showed that the mean operative time during the first year $180 \pm 30 \mathrm{~min}$ and $230 \pm 40 \mathrm{~min}$ and $300 \pm 30$ min during the second and third year.

\section{Conversion Rates}

Eleven out of 178 had a conversion to open approach. Conversion was more often in men and in whom sphincter preservation strategy was desired. Seven out of 11 converted cases $(63.63 \%)$ were amongst men and $36.37 \%$ women $(p=$ 0.04 ) in whom a sphincter preservation was planned. Reasons for conversion are listed in Table 4.

In $18.2 \%$ cases ( 17 of 93 sphincter preservation procedures (ISR excluded)) midline utility incision was utilised for rectal division (after completion of TME).

\section{Histopathologic Outcomes (Table 5)}

Signet cell adenocarcinoma constituted a small volume of 08 $(4.5 \%)$ cases. $56.7 \%(n=101)$ cases were reported to be pT3. Complete pathological response was noted in only $2.8 \%(n=$ 5) cases. Mean distal resection margin length was $2.6 \pm$
Table 3 Operative outcomes

\begin{tabular}{ll}
\hline Variables & Numbers \\
\hline 1. Surgical procedure & 178 \\
a. Abdomino-perineal resection & 78 \\
b. Low anterior resection & 65 \\
c. Anterior resection & 25 \\
d. Intersphincteric resection & 07 \\
e. Posterior exenteration & 03 \\
2. Mean total duration of surgery & $286 \pm 45 \mathrm{~min}$ \\
3. Mean docking time & $13 \pm 5 \mathrm{~min}$ \\
4. Mean surgeon console time & $220 \pm 20 \mathrm{~min}$ \\
5. Mean blood loss & $170 \pm 60 \mathrm{ml}$ \\
6. Conversion rates & $11(6.7 \%)$ \\
\hline
\end{tabular}

$1.8 \mathrm{~cm}$. Two cases $(1.12 \%)$ (one low anterior resection and one intersphincteric resection) had microscopic distal resection margin involvement on final histopathological examination for which a second surgery, APR, was performed on 12th and 15 th postoperative day respectively. $6.74 \%(n=12)$ cases had a positive CRM, and most of these cases had underwent an APR (Fig. 4). Mean number of lymph nodal retrieval was $13 \pm 5$.

\section{Postoperative Complications}

Outcomes of postoperative recovery are presented in Tables 6 and 7. Mean time to first flatus passage was $2 \pm 1$ days; time to resume to oral intake of liquids was $1.5 \pm 0.5$ day. Mean length of hospital stay was $6 \pm 2$ days for APR procedures and $8 \pm 1$ days following sphincter preserving procedures. Urinary catheter removal was performed after 6 days following sphincter preservation procedure and after 8 following APR. Eight/100 (8\%) patients had anastomotic leakage. Twelve/178 (6.74\%) and 10/178 (5.61\%) cases had ClavienDindo grade IIIa and IIIb complications respectively. There was one postoperative death, due to an unexpected cardiac event that occurred on postoperative day 6 following APR.

\section{Discussion}

This study is perhaps the largest series from the Indian subcontinent, and to the best of our knowledge, this is the only Indian series identified in the literature from a regional cancer centre which has analysed short-term oncological outcomes following robotic rectal resection by a robotic novice surgeon.

ALaCaRT and ACOSOG Z6051 randomised trials failed to demonstrate noninferiority of laparoscopic surgery with open surgery for rectal cancer in terms of pathological success, raising concerns about its effect on clinical outcomes. 
Fig. 2 Duration of docking

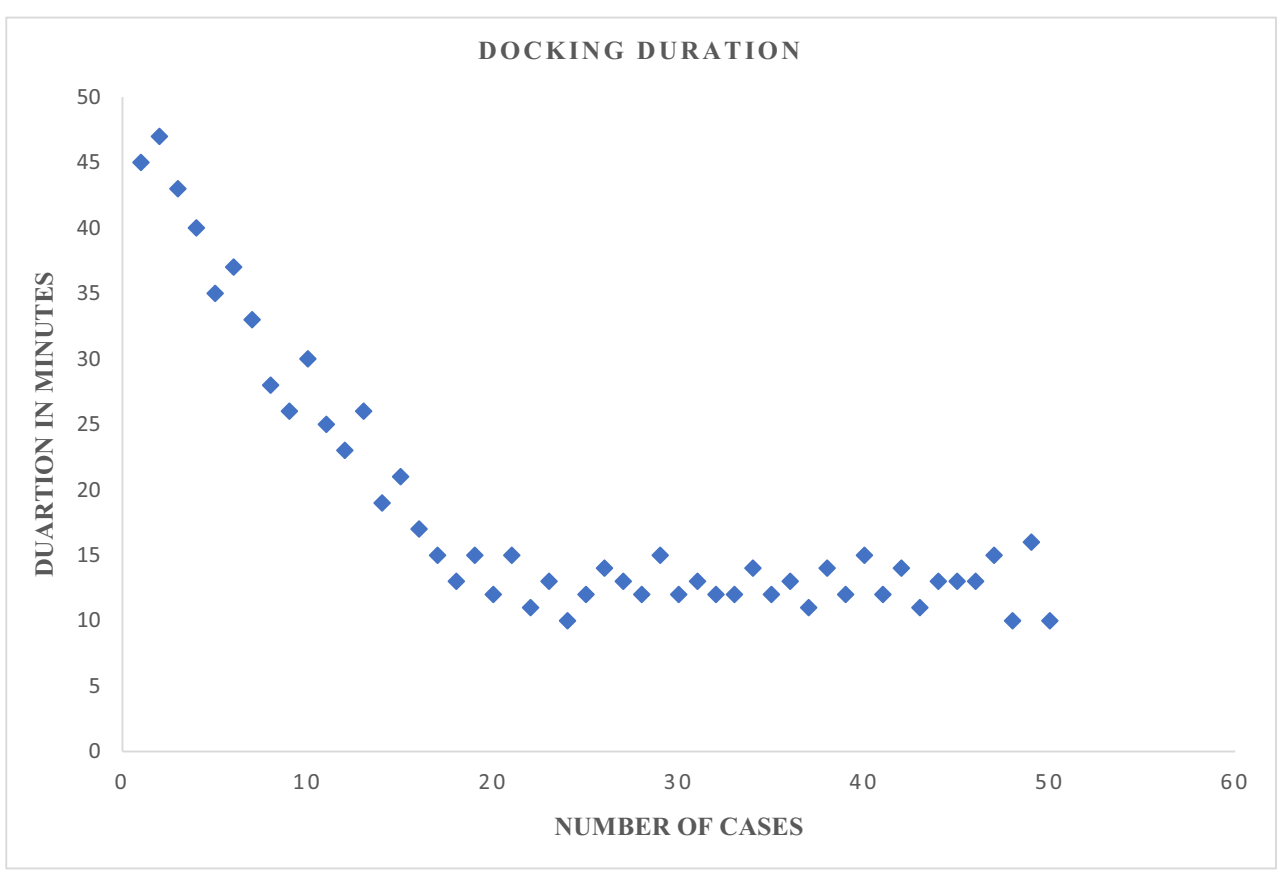

Planned analysis of secondary outcomes after a minimum follow-up of 2 years has not found significant differences in disease-free survival nor locoregional recurrence, although estimates of treatment effect favoured open resection indicating that an alternative platform such as robotics may improve oncological outcomes of minimally invasive surgery. Robotic rectal resection was developed to overcome the limitations of conventional laparoscopy and to achieve a superior quality of oncologic resection [30].

As a new surgical procedure, worldwide R-TME is adopted increasingly by surgeons. Patient selection is of utmost importance during the initial period of learning. In our series, all three surgeons were making a transition from open to robotic approach and were novice with laparoscopic approach; hence during the initial year, the surgeons selected cases suitable for robotic APR which were less complex until more experience was gained; this explains our APR rates of 43.82\% (78/178) cases. During the first year, the ratio of APR to sphincter preserving procedures was $4: 1$; with increasingly gaining experience, the ratio was $2: 2$ during the first half of the second year and 1:4 thereafter.

Existing literature demonstrate longer operative time for robotics compared with open and laparoscopic rectal resections [31-34]. ROLARR trial reported a mean duration of
Fig. 3 Annual case selection distribution

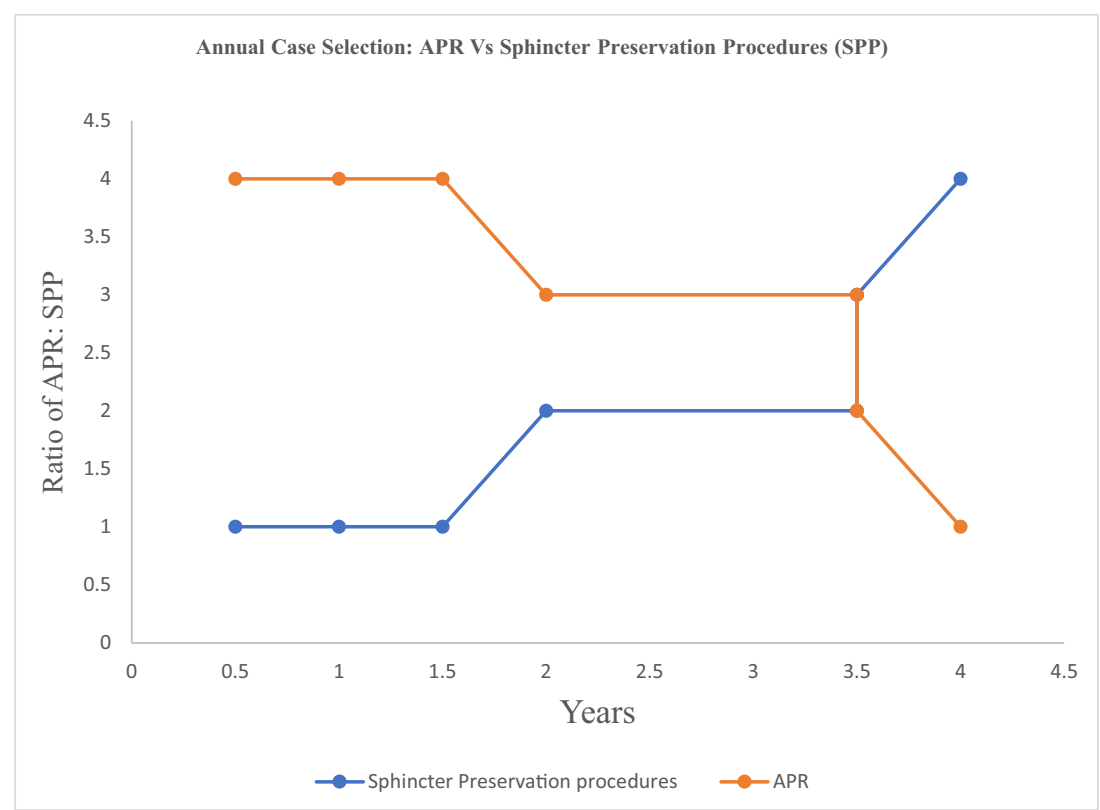


Table 4 Factors favouring conversion to open approach

\begin{tabular}{ll}
\hline Unfavourable parameters & Numbers $(n=11)$ \\
\hline 1. Uncontrolled bleeding from Inferior mesenteric artery & 01 \\
2. Hem-o-lock clip slippage from inferior mesenteric artery stump & 01 \\
3. Common iliac artery bleeding while dissecting the left ureter & 01 \\
4. Peri-hilar bleeding while splenic flexure mobilisation & 01 \\
5. Descending colon perforation while mobilising splenic flexure & 01 \\
6. Pre-sacral venous plexus injury and bleed & 01 \\
7. Rectal perforation while dividing meso-rectum & 01 \\
8. Rectal disruption while insertion of stapler & 02 \\
9. Iatrogenic tumour perforation & 01 \\
10. Incidentally detected multiple bulky lateral pelvic lymph nodes & 01 \\
\hline
\end{tabular}

surgery of $298.5 \pm 88.71$. Mean operative time in our series is $286 \pm 45 \mathrm{~min}$. Further, an annual analysis of cases showed that the mean operative time during the first year $180 \pm 30 \mathrm{~min}$ and $230 \pm 40 \mathrm{~min}$ and $300 \pm 30 \mathrm{~min}$ during the second and third year. This progressively increasing annual operative time is due to routine splenic flexure mobilisation and more number

Table 5 Histopathologic outcomes

\begin{tabular}{ll}
\hline Variables & Numbers $(\%)$ \\
\hline 1. Histopathology & $n=178$ \\
a. Adenocarcinoma & $138(78.4 \%)$ \\
b. Mucinous adenocarcinoma & $30(17.0 \%)$ \\
c. Signet ring adenocarcinoma & $08(4.5 \%)$ \\
d. Melanoma & $02(1.12 \%)$ \\
2. Grade & $n=176$ \\
a. I & $51(28.7 \%)$ \\
b. II & $87(48.8 \%)$ \\
c. III & $38(22.4 \%)$ \\
3. Pathological T stage & \\
a. pT1 & $9(5.1 \%)$ \\
b. pT2 & $63(35.4 \%)$ \\
c. pT3 & $101(56.7 \%)$ \\
d. CPR & $5(2.8 \%)$ \\
4. Pathological nodal stage & \\
a. N0 & $96(53.9 \%)$ \\
b. N1 & $43(24.1 \%)$ \\
c. N2 & $39(21.9 \%)$ \\
5. Distal resection margin status & \\
a. Negative & $176(98.8 \%)$ \\
b. Positive & $02(1.12 \%)$ \\
6. Mean distal resection margin length & $2.6 \pm 1.8 \mathrm{cms}$ \\
7. Circumferential resection margin status & \\
a. Negative & $166(93.25 \%)$ \\
b. Positive & $12(6.74 \%)$ \\
8. Mean number of lymph nodal yield & $13 \pm 5$ \\
\hline
\end{tabular}

$C P R$ Complete pathological response of sphincter preservation procedures which are been performed from second year onwards.

The landmark laparoscopic rectal cancer trials, MRC CLASICC, COLOR-II, ACOSOG-Z6051, ALaCaRT reported a conversion rates of $34 \%, 16 \%, 11 \%$ and $9 \%$ respectively. The conversion rates reported in ROLARR trial is $12.2 \%$ in the laparoscopic group and $8.1 \%$ in the robotic-assisted group. The present series reports a conversion rate of $6.7 \%(11 / 178)$. Table 4 illustrates the reasons for conversion, and conversion rates were more often in men and when the intended procedure was sphincter preservation procedure as compared with APR. In 5/11, failure to progress into the pelvis was noted and more often in the initial few cases. Indeed, the rate of conversion in the current series is lower than reported in ROLARR but consistent with other reports from experienced centres [35]. Optimal preoperative imaging, evaluation and case selection are the attributable factors for the low conversion rates.

Robotic approach seems to facilitate mesorectal dissection, particularly in mid and low rectal tumours, hence one of the major benefit thought to be conferred by this novel approach was lower positive CRM rates. Our evaluation of CRM positivity and DRM involvement assessed the quality of mesorectal excision. Our study reports 12/178 (6.74\%) CRM positive rates and 2/178 (1.12\%) DRM involvement.

CRM positive was more common amongst patients who had underwent APR (Fig. 3). Ten/12 and 2/12 patients with positive CRM had underwent APR and low AR respectively. Seven/10 APR patients with positive CRM had persistent MRF involvement despite NACRT and additional FOLFOX $\times 4$. Three/10 APR had positive CRM secondary to tumour perforation. One patient with DRM involvement following intersphincteric resection (ISR) underwent completion APR; the other ISR patient did not consent for APR and is on follow-up. Our CRM positive rates are slightly higher than ROLARR trial, reported to be $5.1 \%$; this is probably attributed to undertaking APR procedures for mucinous or signet ring adenocarcinoma with persistently MRF positive despite NACRT and additional chemotherapy. The response to neoadjuvant treatment in this histology group is nonfavourable [36, 37]. 
Fig. 4 Consort of positive CRM cases

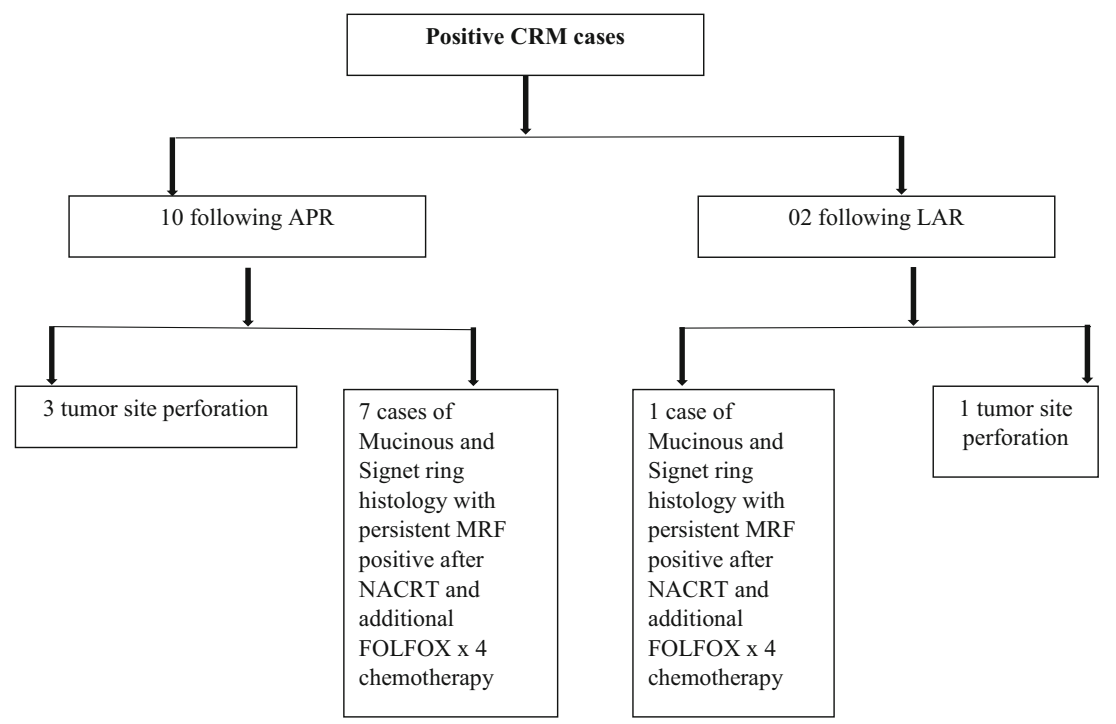

The mean time to fist passage of flatus resume to oral liquid intake $2 \pm 1$ and $1.5 \pm 0.5$ days, respectively. Median length of hospitalisation was 7 days (range 4-14 days). Further gains in perioperative care and reduced length of stay may be possible by standardization of enhanced recovery after surgery programs. The overall complications $(\mathrm{CD}$ grade $\geq 2)$ reported in the present review is $29.76 \%$. In the present study, major complications requiring radiologic intervention or surgical treatment were included in CD grade IIIa-IIIb. Of the 100 patients with an anastomosis, $8(8 \%)$ cases had a leak. All the 8 cases required interventions as listed in Table 7 . ROLARR trial reports overall 30-day complication and anastomotic leak rates of $33.1 \%$ and $12.2 \%$ respectively.

Although the results of this study are comparable with those of the ROLARR trial, there exist significant differences

Table 6 Postoperative outcomes and complications

\begin{tabular}{ll}
\hline Parameters & Numbers (\%) \\
\hline 1. Mean time to first passage of flatus (days) & $2 \pm 1$ \\
2. Mean time to resume to oral intake of liquids (days) & $1.5 \pm 0.5$ \\
3. Mean length of hospital stay (days) & \\
a. APR & $6 \pm 2$ \\
b. Sphincter preservation R-TME & $8 \pm 1$ \\
4. Anastomotic leak rate & $8(8 \%)$ \\
5. Clavein-Dindo complications & \\
a. Grade I & $125(70.22 \%)$ \\
b. Grade II & $29(16.29 \%)$ \\
c. Grade IIIa & $12(6.74 \%)$ \\
d. Grade IIIb & $10(5.61 \%)$ \\
e. Grade IV & $01(0.56 \%)$ \\
f. Grade V & $01(0.56 \%)$ \\
\hline
\end{tabular}

$A P R$ Abdomino-perineal resection, $R-T M E$ robotic total mesorectal excision in the case selection and types of procedures between these two studies.

Our study results are in a good agreement with that reported by ROLARR group in terms of perioperative and pathological outcomes; however, this study has various limitations such as its retrospective nature, lack of data on bladder and sexual function, which may have reflected the quality of rectal dissection.

\section{Conclusion}

In conclusion, perioperative and pathologic outcomes following robotic rectal resection reflect that it is safe, effective and reproducible by a minimally invasive novice surgeon. Careful patient selection, choosing less complex procedures and standardised protocol during the initial cases is of fundamental importance for a surgeon making a transition from open to

Table 7 Interventions for anastomotic leak

\begin{tabular}{ll}
\hline Procedures & Interventions \\
\hline Two cases of low AR without DS & $\begin{array}{c}\text { Exploration followed by peritoneal } \\
\text { lavage and loop ileostomy } \\
\text { Laparoscopic peritoneal lavage in } \\
\text { view of minimal pelvic confined } \\
\text { contamination }\end{array}$ \\
$\begin{array}{c}\text { Drainage of the pelvic collection } \\
\text { anastomotic dehiscence } \\
\text { following ISR }\end{array}$ & $\begin{array}{l}\text { parth trans anal repair of the } \\
\text { site }\end{array}$ \\
$\begin{array}{c}\text { Low rectovaginal fistula following } \\
\text { low AR }\end{array}$ & $\begin{array}{c}\text { Completion APR } \\
\text { Two cases of low AR with DS in } \\
\text { hemodynamically stable patients }\end{array}$ \\
Radiologically guided pigtail \\
drainage of collection
\end{tabular}

$A R$ Anterior resection, ISR intersphincteric resection, $D S$ diversion stoma, $A P R$ abdomino-perineal resection 
robotic approach. Future studies are required to determine long-term oncologic and functional outcomes.

\section{Compliance with Ethical Standards}

Conflict of Interest The authors declare that they have no conflict of interest.

Ethics Approval The study protocol conforms to the ethical guidelines of the World Medical Association Declaration of Helsinki: Ethical Principles for Medical Research Involving Human Subjects adopted by the 18th WMA General Assembly, Helsinki, Finland, June 1964, as revised in Tokyo 2004. No approval of the institutional review committee was needed.

Consent to Participate All patients had consented for the proposed surgery. This is a retrospective analysis of prospectively maintained database which does not necessitate consenting for analysis.

Consent for Publications All authors have consented for publication.

Open Access This article is licensed under a Creative Commons Attribution 4.0 International License, which permits use, sharing, adaptation, distribution and reproduction in any medium or format, as long as you give appropriate credit to the original author(s) and the source, provide a link to the Creative Commons licence, and indicate if changes were made. The images or other third party material in this article are included in the article's Creative Commons licence, unless indicated otherwise in a credit line to the material. If material is not included in the article's Creative Commons licence and your intended use is not permitted by statutory regulation or exceeds the permitted use, you will need to obtain permission directly from the copyright holder. To view a copy of this licence, visit http://creativecommons.org/licenses/by/4.0/.

\section{References}

1. De Caluwe L, Van Nieuwenhove Y, Ceelen WP (2013) Preoperative chemoradiation versus radiation alone for stage II and III resectable rectal cancer. Cochrane Database Syst Rev 2: CD006041. https://doi.org/10.1002/14651858

2. Heald RJ, Husband EM, Ryall RD (1982) The mesorectum in rectal cancer surgery-the clue to pelvic recurrence? Br J Surg 69:613616. https://doi.org/10.1002/bjs. 1800691019

3. Quirke P, Steele R, Monson J, Grieve R, Khanna S, Couture J, O'Callaghan C, Myint AS, Bessell E, Thompson LC, Parmar M, Stephens RJ, Sebag-Montefiore D, MRC CR07/NCIC-CTG CO16 Trial Investigators, NCRI Colorectal Cancer Study Group (2009) Effect of the plane of surgery achieved on local recurrence in patients with operable rectal cancer: a prospective study using data from the MRC CR07 and NCIC-CTG CO16 randomised clinical trial. Lancet 373:821-828. https://doi.org/10.1016/S0140-6736(09) 60485-2

4. Leonard D, Penninckx F, Laenen A, Kartheuser A (2015) Procare. Scoring the quality of total mesorectal excision for the prediction of cancer-specific outcome. Color Dis 17:O115-O122. https://doi.org/ 10.1111/codi.12931

5. Kitz J, Fokas E, Beissbarth T, Strobel P, Wittekind C et al (2018) Association of plane of total mesorectal excision with prognosis of rectal cancer: secondary analysis of the CAO/ARO/AIO-04 phase 3 randomized clinical trial. JAMA Surg 153:181607. https://doi.org/ 10.1001/jamasurg.2018.1607
6. Kusters M, Marijnen CA, van de Velde CJ, Rutten HJ, Lahaye MJ et al (2010) Patterns of local recurrence in rectal cancer; a study of the Dutch TME trial. Eur J Surg Oncol 36:470-476. https://doi.org/ 10.1016/j.ejso.2009.11.011

7. Nagtegaal ID, Quirke P (2008) What is the role for the circumferential margin in the modern treatment of rectal cancer? J Clin Oncol 26:303-312. https://doi.org/10.1200/JCO.2007.12.7027

8. Birbeck KF, Macklin CP, Tiffin NJ, Parsons W, Dixon MF, Mapstone NP, Abbott CR, Scott N, Finan PJ, Johnston D, Quirke P (2002) Rates of circumferential resection margin involvement vary between surgeons and predict outcomes in rectal cancer surgery. Ann Surg 235:449-457. https://doi.org/10.1097/00000658200204000-00001

9. Garcia-Granero E, Faiz O, Munoz E, Flor B, Navarro S et al (2009) Macroscopic assessment of mesorectal excision in rectal cancer: a useful tool for improving quality control in a multidisciplinary team. Cancer 115:3400-3411. https://doi.org/10.1002/cncr.24387

10. Jayne DG, Guillou PJ, Thorpe H, Quirke P, Copeland J, Smith AM, Heath RM, Brown JM, Group UMCT (2007) Randomized trial of laparoscopic-assisted resection of colorectal carcinoma: 3-year results of the UK MRC CLASICC trial group. J Clin Oncol 25(21): 3061-3068

11. Kang SB, Park JW, Jeong SY et al (2010) Open versus laparoscopic surgery for mid or low rectal cancer after neoadjuvant chemoradiotherapy (COREAN trial): short-term outcomes of an open-label randomised controlled trial. Lancet Oncol 11(7):637-645. https:// doi.org/10.1016/S1470-2045(10)70131-5

12. Van der Pas MH, Haglind E, Cuesta MA, Furst A, Lacy AM, Hop WC, Bonjer HJ, COlorectal cancer Laparoscopic or Open Resection II (COLOR II) Study Group (2013) Laparoscopic versus open surgery for rectal cancer (COLOR II): short-term outcomes of a randomised, phase 3 trial. Lancet Oncol 14(3):210-218. https:// doi.org/10.1016/S1470-2045(13)70016-0

13. Kitano S, Inomata M, Sato A, Yoshimura K, Moriya Y, Japan Clinical Oncology Group Study (2005) Randomized controlled trial to evaluate laparoscopic surgery for colorectal cancer: Japan clinical oncology group study JCOG 0404. Jpn J Clin Oncol 35(14): 1324-1332. https://doi.org/10.1093/jjco/hyi124

14. Bonjer HJ, Deijen CL, Haglind E, COLOR II Study Group (2015) A randomized trial of laparoscopic versus open surgery for rectal cancer. N Engl J Med 372(14):1324-1332. https://doi.org/10.1056/ NEJMoa1414882

15. Fleshman J, Branda M, Sargent DJ, Boller AM, George V, Abbas M, Peters WR Jr, Maun D, Chang G, Herline A, Fichera A, Mutch M, Wexner S, Whiteford M, Marks J, Birnbaum E, Margolin D, Larson D, Marcello P, Posner M, Read T, Monson J, Wren SM, Pisters PWT, Nelson H (2015) Effect of laparoscopic-assisted resection vs open resection of stage II or III rectal cancer on pathologic outcomes: the ACOSOG Z6051 randomized clinical trial. JAMA 314:1346-1355. https://doi.org/10.1001/jama.2015.10529

16. Stevenson AR, Solomon MJ, Lumley JW, Hewett P, Clouston AD, Gebski VJ, Davies L, Wilson K, Hague W, Simes J, ALaCaRT Investigators (2015) Effect of laparoscopic-assisted resection vs open resection on pathological outcomes in rectal cancer: the ALaCaRT randomized clinical trial. JAMA 314:1356-1363. https://doi.org/10.1001/jama.2015.12009

17. Martinez-Perez A, Carra MC, Brunetti F, de'Angelis N. (2017) Pathologic outcomes of laparoscopic vs open mesorectal excision for rectal cancer: a systematic review and meta-analysis. JAMA Surg 152:e165665. https://doi.org/10.1001/jamasurg.2016.5665

18. Fleshman J, Branda ME, Sargent DJ, Boller AM, George VV et al (2019) Disease-free survival and local recurrence for laparoscopic resection compared with open resection of stage II to III rectal cancer: follow-up results of the ACOSOG Z6051 randomized controlled trial. Ann Surg 269:589-595. https://doi.org/10.1097/SLA. 0000000000003002 
19. Stevenson ARL, Solomon MJ, Brown CSB, Lumley JW, Hewett P, Clouston AD, Gebski VJ, Wilson K, Hague W, Simes J, Australasian Gastro-Intestinal Trials Group (AGITG) ALaCaRT investigators (2019) Disease-free survival and local recurrence after laparoscopic assisted resection or open resection for rectal cancer: the australasian laparoscopic cancer of the rectum randomized clinical trial. Ann Surg 269:596-602. https://doi.org/10.1097/SLA. 0000000000003021

20. Martinez-Perez A, de'Angelis N. (2019) Comment on "Mid-term results of ACOSOG Z6051 trial sustain the unresolved debate". Ann Surg 270:e52-e53

21. Baek SJ, Kim CH, Cho MS et al (2015) Robotic surgery for rectal cancer can overcome difficulties associated with pelvic anatomy. Surg Endosc 29:1419-1424. https://doi.org/10.1007/s00464-0143818-x

22. Allemann P, Duvoisin C, Di Mare L et al (2016) Robotic-assisted surgery improves the quality of total mesorectal excision for rectal cancer compared to laparoscopy: results of a case-controlled analysis. World J Surg 40:1010-1016

23. Kang J, Yoon KJ, Min BS, Hur H, Baik SH, Kim NK, Lee KY (2013) The impact of robotic surgery for mid and low rectal cancer: a case-matched analysis of a 3-arm comparison-open, laparoscopic, and robotic surgery. Ann Surg 257:95-101. https://doi.org/10. 1097/SLA.0b013e3182686bbd

24. Baik SH, Ko YT, Kang CM, Lee WJ, Kim NK, Sohn SK, Chi HS, Cho CH (2008) Robotic tumor-specific mesorectal excision of rectal cancer: short-term outcome of a pilot randomized trial. Surg Endosc 22:1601-1608. https://doi.org/10.1007/s00464-008-9752-z

25. Jayne D, Pigazzi A, Marshall H, Croft J, Corrigan N, Copeland J, Quirke P, West N, Rautio T, Thomassen N, Tilney H, Gudgeon M, Bianchi PP, Edlin R, Hulme C, Brown J (2017) Effect of roboticassisted vs conventional laparoscopic surgery on risk of conversion to open laparotomy among patients undergoing resection for rectal cancer: the ROLARR randomized clinical trial. Jama 318:15691580. https://doi.org/10.1001/jama.2017.7219

26. Glynne-Jones R, Wyrwicz L, Tiret E, Brown et al (2017) Rectal cancer: ESMO clinical practice guidelines for diagnosis, treatment and follow-up. Ann Oncol 28(suppl_4):iv22-iv40. https://doi.org/ 10.1093/annonc/mdx224

27. Nagtegaal ID, van de Velde CJ, van der Worp E et al (2002) Macroscopic evaluation of rectal cancer resection specimen: clinical significance of the pathologist in quality control. J Clin Oncol 20:1729-1734. https://doi.org/10.1200/JCO.2002.07.010

28. Tamhankar AS, Jatal S, Saklani A (2016) Total robotic radical rectal resection with Da Vinci Xi system: single docking, single phase technique. Int J Med Robot 12:642-647. https://doi.org/10. 1002/rcs. 1734

29. Clavien PA, Barkun, et al. (2009) The Clavien-Dindo classification of surgical complications: five-year experience. Ann Surg 250: 187-196. https://doi.org/10.1097/SLA.0b013e3181b13ca2

30. Wexner SD, Bergamaschi R, Lacy A et al (2009) The current status of robotic pelvic surgery: results of a multinational interdisciplinary consensus conference. Surg Endosc 23:438-443. https://doi.org/10. 1007/s10151-018-1766-5

31. Prete FP, Pezzolla A, Prete F, Testini M, Marzaioli R, Patriti A, Jimenez-Rodriguez RM, Gurrado A, Strippoli GFM (2018) Robotic versus laparoscopic minimally invasive surgery for rectal cancer: a systematic review and meta-analysis of randomized controlled trials. Ann Surg 267:1034-1046. https://doi.org/10.1097/ SLA.0000000000002523

32. Ohtani H, Maeda K, Nomura S, Shinto O, Mizuyama Y et al (2018) Meta-analysis of robot-assisted versus laparoscopic surgery for rectal cancer. In Vivo 32:611-623. https://doi.org/10.21873/invivo. 11283

33. Lee SH, Lim S, Kim JH, Lee KY (2015) Robotic versus conventional laparoscopic surgery for rectal cancer: systematic review and metaanalysis. Ann Surg Treat Res 89:190-201. https://doi.org/10. 4174/astr.2015.89.4.190

34. Somashekhar SP, Ashwin KR, Rajashekhar J, Zaveri S (2015) Prospective randomized study comparing robotic-assisted surgery with traditional laparotomy for rectal cancer-Indian study. Indian J Surg 77:788-794. https://doi.org/10.1007/s12262-013-1003-4

35. Baik SH, Kim NK, Lim DR, Hur H, Min BS, Lee KY (2013) Oncologic outcomes and perioperative clinicopathologic results after robot-assisted tumor-specific mesorectal excision for rectal cancer. Ann Surg Oncol 20:2625-2632. https://doi.org/10.1245/ s10434-013-2895-8

36. Shinde RS, Katdare N, Kumar NAN, Bhamre R, Desouza A, Ostwal V, Engineer R, Saklani A (2018) Impact of histological subtype on treatment outcomes in locally advanced rectal adenocarcinoma treated with neoadjuvant chemoradiation. Acta Oncol 57(12):1721-1723. https://doi.org/10.1080/0284186X.2018. 1502893

37. Yu SK, Chand M, Tait DM et al (2014) Magnetic resonance imaging defined mucinous rectal carcinoma is an independent imaging biomarker for poor prognosis and poor response to preoperative chemoradiotherapy. Eur J Cancer 50:920-927

Publisher's Note Springer Nature remains neutral with regard to jurisdictional claims in published maps and institutional affiliations. 